حامد نوذرى' و فاطمه توكلى

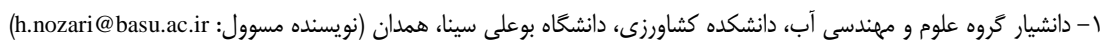

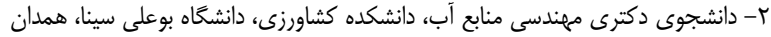

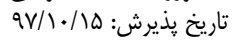

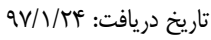

صفحه: ا تا با

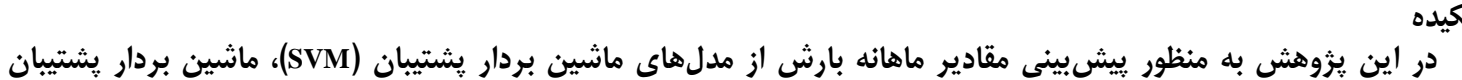

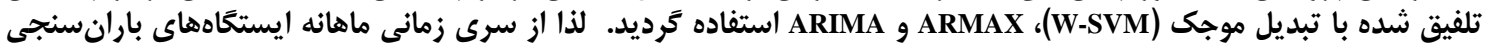

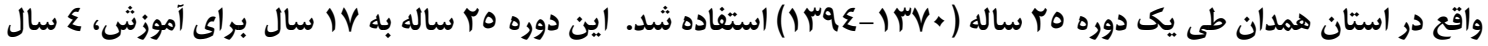

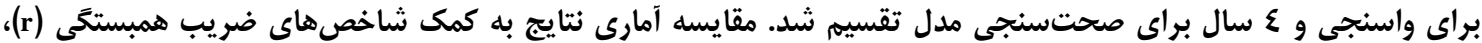

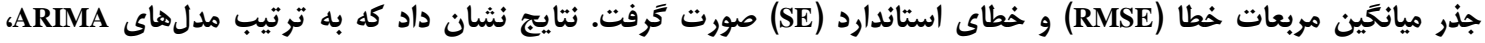

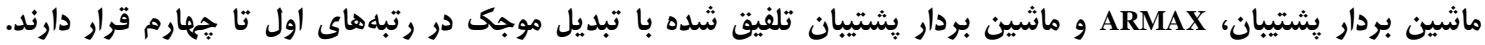

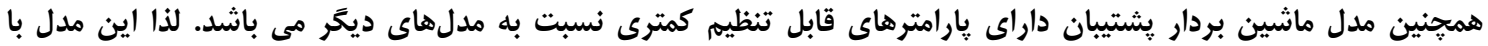

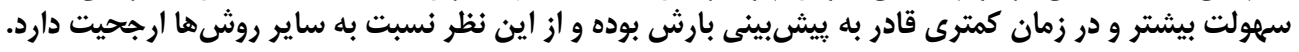

وازههاى كليدى: ARIMA، ARMAX، بارش، بيشبينى، ماشين بردار بشتيبان، موجك

درجه حرارت مشابه با شبكه عصبى MLP رفتار نموده است

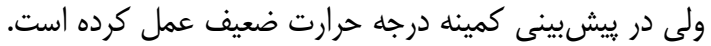

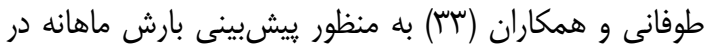

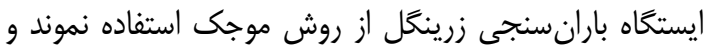

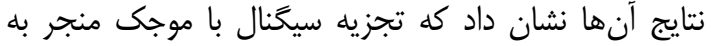

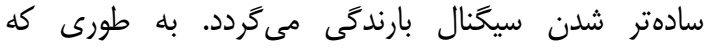

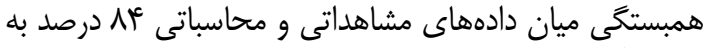

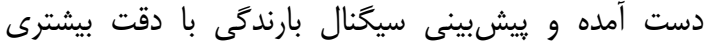
صورت گرفته است.

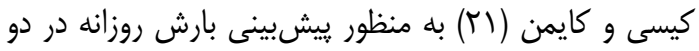

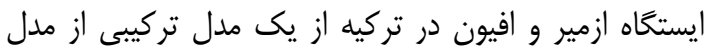

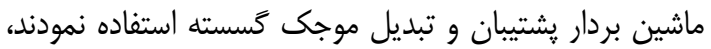

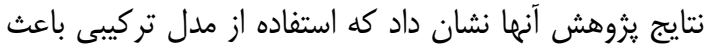

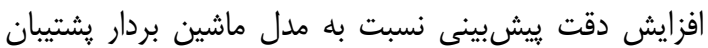
مستقل زرديد.

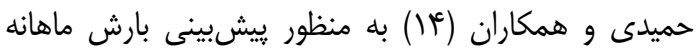

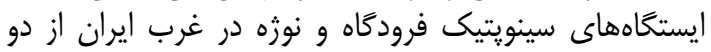

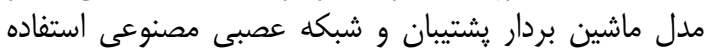

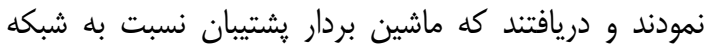
عصبى از كارايى بيشترى برخوردار است.

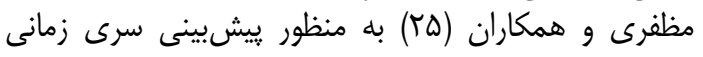

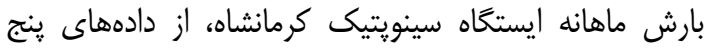

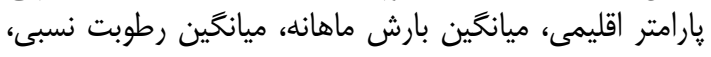

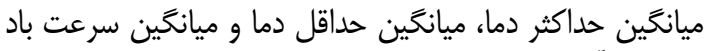

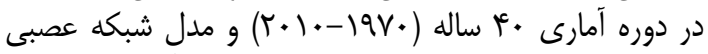

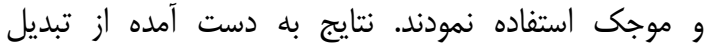

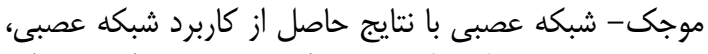

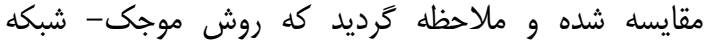

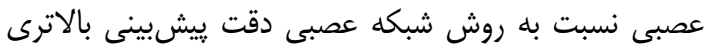

بارش به عنوان يك عنصر مهام از منابع آبى در تمام

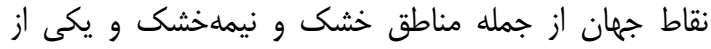

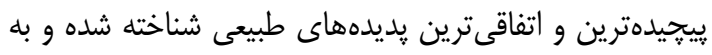

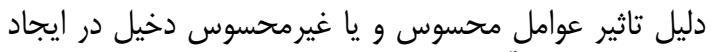

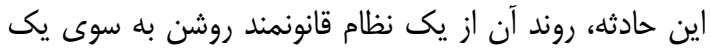

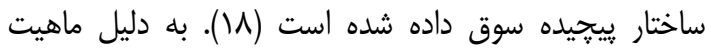
غيرخطى، عدم قطعيت، عدم صراحت زيلت زياد و ويثز

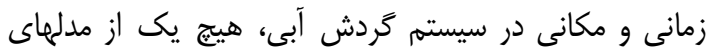

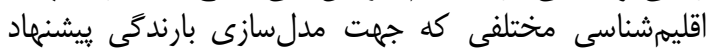

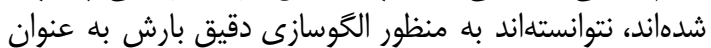

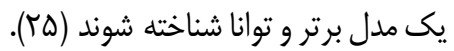

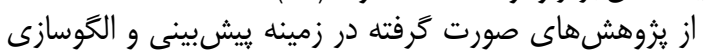

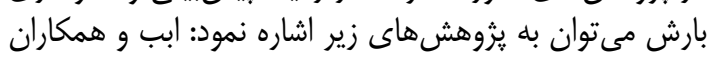

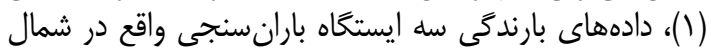

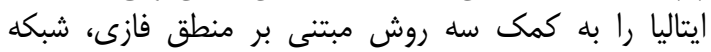

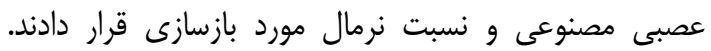

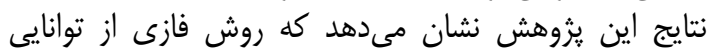

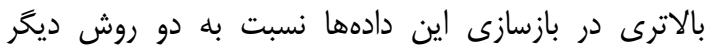
برخوردار است.

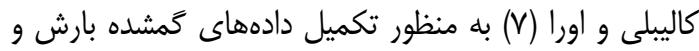

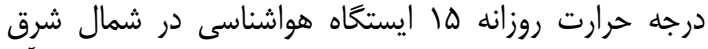

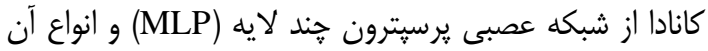

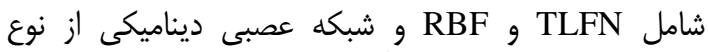
بازگشتى (RNN) و انواع آن شامل

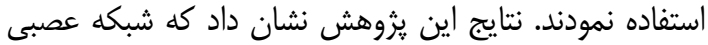

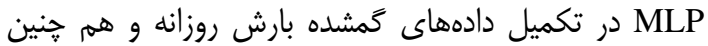

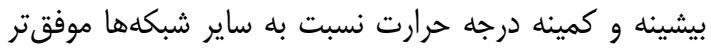

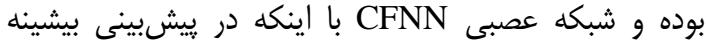


مصنوعى و مدل SARIMA صورت گرفته و در نهايت نتايج

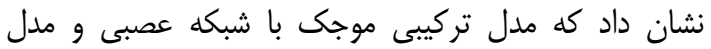

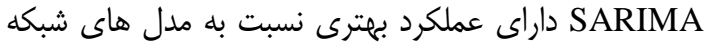
عصبى و SARIMA در ييشبينى بارش است.

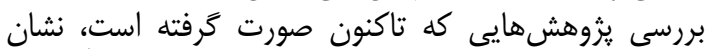

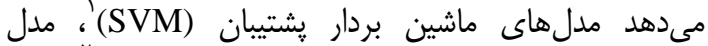

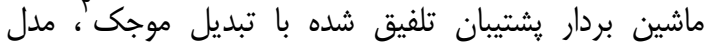
ARMAX (ARIMA)

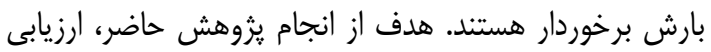

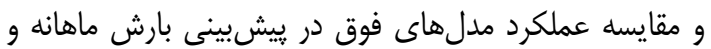

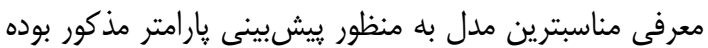

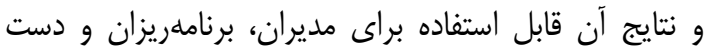

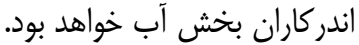

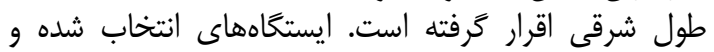

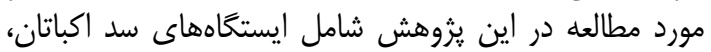

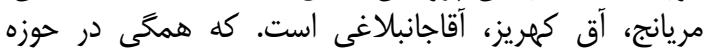

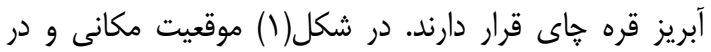
جداول (I) و (Y) مشخصات اين ايستخاهها نشان داده شده

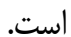

دارد و همجنين دقت ييشبينى در هر دو مدل با افزايش تعداد

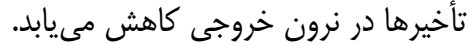

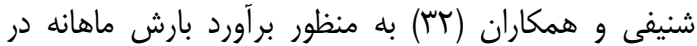
SERBIA

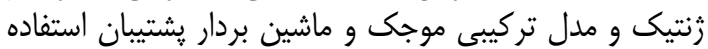

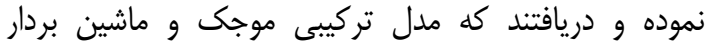

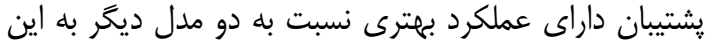

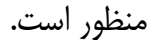

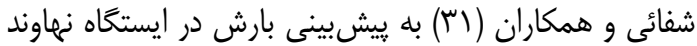

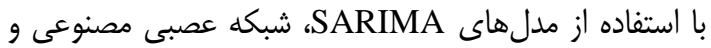

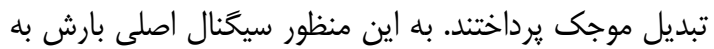

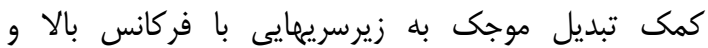

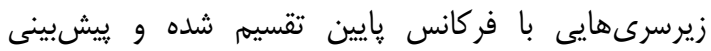

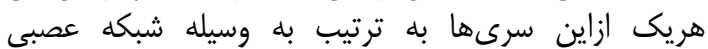

\section{مواد و روشها منطقه مورد مطالعه}

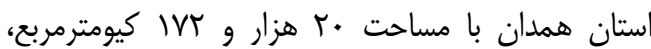

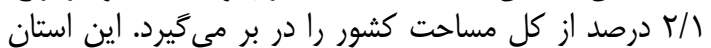

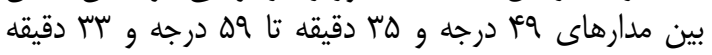

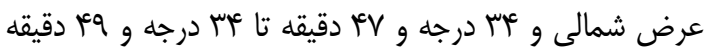

Table 1. Characteristics of studied rain gauge stations

جدول ا- مشخصات ايستخاههاى باران سنجى مورد مطالعه

\begin{tabular}{|c|c|c|c|c|c|}
\hline إرتفاع) & 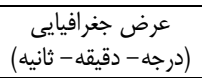 & 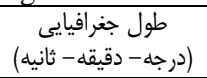 & نوع ايستخاه & نام ايستگاه & رديف \\
\hline $190 \mathrm{~V}$ & $\mu \varphi-\varphi \Delta-\Gamma \varphi$ & $11-r g-r \lambda$ & باران سنج ثبات & سد اكباتان & 1 \\
\hline M.Y & $\Delta \Gamma-\Delta \cdot-M r$ & $\cdot v-\cdot r-r \wedge$ & باران سنج معمولى & آقاجانبالاغى & r \\
\hline Whe & $F \cdot-\Delta q-\mu F$ & $\cdot \mid-r \cdot-r \wedge$ & باران سنج ثبات & آق كهريز & $r$ \\
\hline$|A+|$ & $p \mid-p q-r q \varepsilon$ & $r \Lambda-r V-r \Lambda$ & باران سنج معمولى & مريانج & f \\
\hline
\end{tabular}

Table 2. Characteristics of studied rain gauge stations

\begin{tabular}{|c|c|c|c|c|c|}
\hline $\begin{array}{l}\text { متوسط بارش/month) } \\
\text { (mm/mo }\end{array}$ & 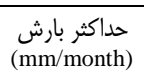 & حداقل بارش & طول دوره أمارى & نام ايستخاه & رديف \\
\hline TN/V & $19 \mathrm{~V}$ & . & هesm & سد اكباتان & 1 \\
\hline$r q / r$ & $198 / 0$ & . & هes & آقاجانبلاغى & r \\
\hline TV & IAV & . & $\mid r q q-1 f^{2} q$ & آق كهريز & r \\
\hline
\end{tabular}




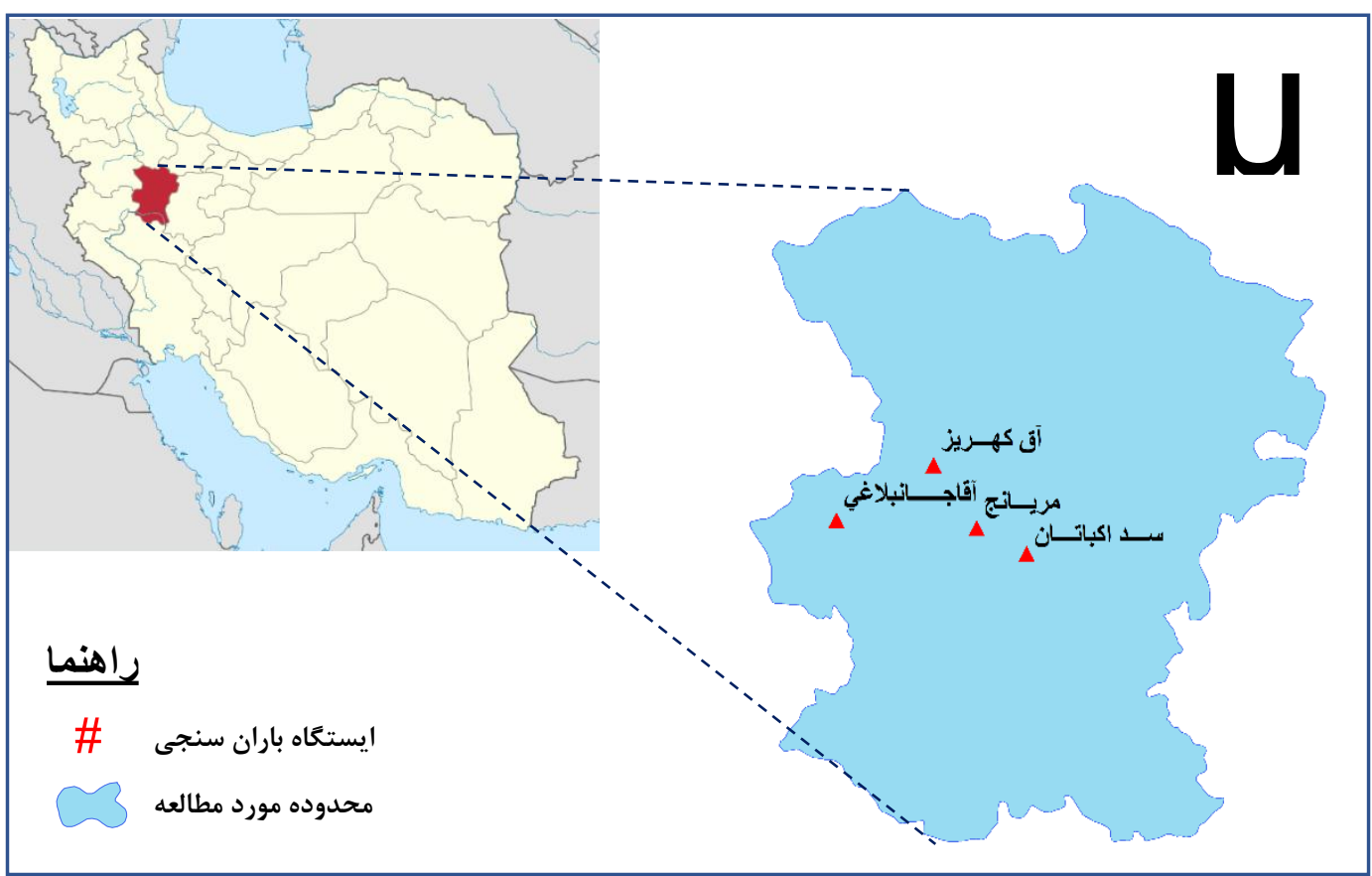

شكل (- موقعيت مكانى ايستخامهاى باران سنجى مورد مطالعه

Figure 1. Location of the studied rain gauge stations

دو ويزگى برجسته قابليت عمومى سازى عالى و سازكارى باري

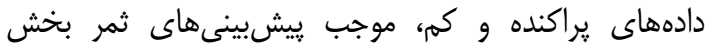

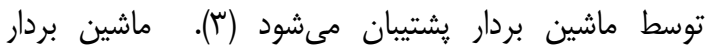

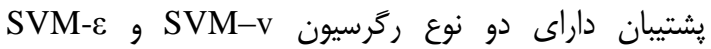

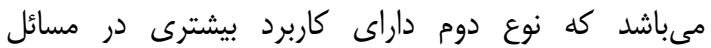

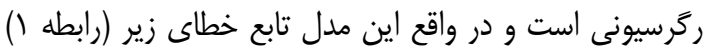

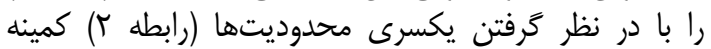

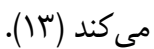

$\frac{1}{2} W^{T} W+C \sum_{i=1}^{N} \xi_{i}+C \sum_{i=1}^{N} \xi_{i}^{*}$

$W^{T} \phi\left(x_{i}\right)+b-y_{i} \leq \varepsilon+\xi_{i}^{*}$

$y_{i}-W^{T} \phi\left(x_{i}\right)-b \leq \varepsilon+\xi_{i}^{*}$

$\xi_{i}^{*}, \xi_{i} \geq 0$

تبديل موجك عبارت است از عملياتى كه از تغيير در توابع

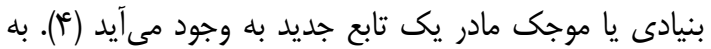

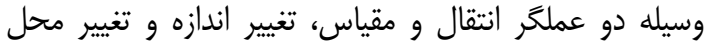

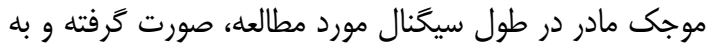
صورت زير (رابطه بّ) بيان مى شود (·r).

$\Psi_{\mathrm{a}, \mathrm{b}}(\mathrm{t})=|\mathrm{a}|^{-1 / 2} \Psi\left(\frac{\mathrm{t}-\mathrm{a}}{\mathrm{b}}\right)$,

$\mathrm{b} \in \mathrm{R}, \mathrm{a} \in \mathrm{R}, \mathrm{a} \neq 0$

هر موجى داراى سه مشخصه است كه شرط مقبوليت نيز ناميده شده و عبارتند از تعداد نوسان محدود، باز گشت سريع بـ به به ني
مدل ماشين بردار يشتيبان

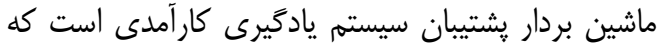

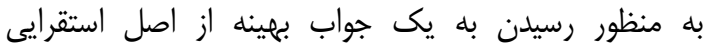

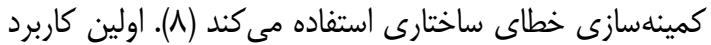

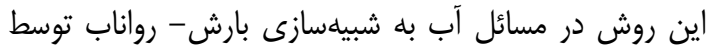

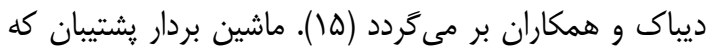

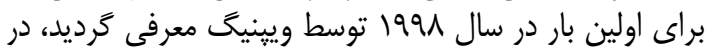
واقع يك طبقه كننده دو دويى است (عال).

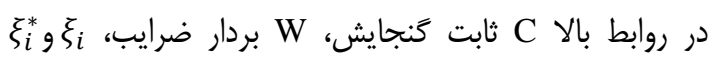

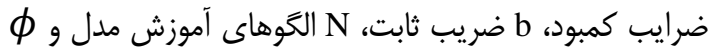
تابع كرنل است.

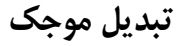

در اين رابطه a پارامتر مقياس يا فر كانس، b بارامتر انتقال يا زمان و R محدوده اعداد حقيقى است. 
سيكنالهاى زمانى كه در اختيار هيدرولوزيستها قرار دارند،

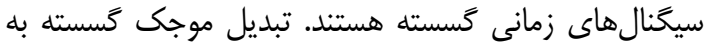
صورت زير تعريف مى گردد (بان): $W_{f}(a, b)=|a|^{\frac{-1}{2} \Delta t} \sum_{k=1}^{N} f(k \Delta t) \Psi^{*}\left(\frac{k \Delta t-b}{a}\right)$

مدل ARMAX مدل ARMAX يك حالت توسعه يافته از مدل آرما بوده كه

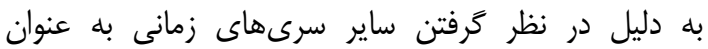

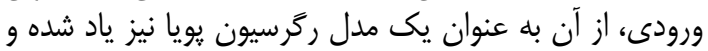
به صورت زير قابل تعريف است (سٓ).

$$
\begin{gathered}
y_{t}=c+\sum_{i=1}^{p} \varphi_{i} y_{t-i}+\varepsilon_{t}+\sum_{j=1}^{q} \phi_{j} \varepsilon_{t-j} \\
+\sum_{k=1}^{n} \beta_{k} x(t, k)
\end{gathered}
$$

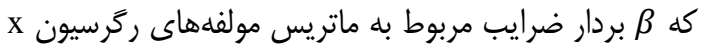

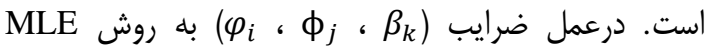
تخمين زده مىشوند.

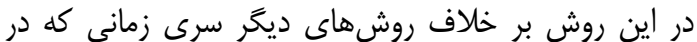

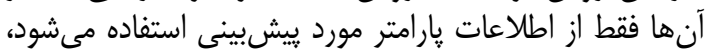

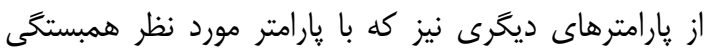

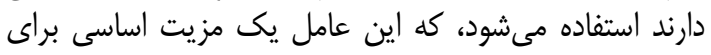

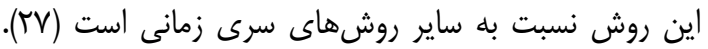

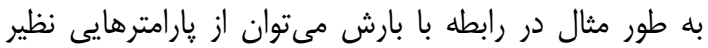

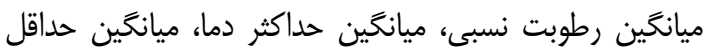

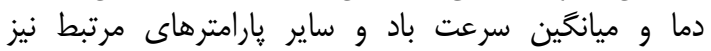

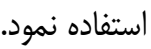

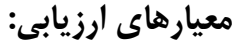
به منظور ارزيابى كارايى و دقت مدلهائ مارئ مورد استفاده در

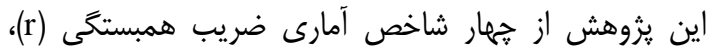

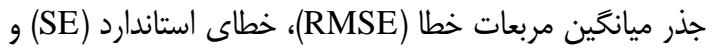
معيار اطلاعات (AIC) استفاده شد.

$r=\frac{\left[\sum_{i=1}^{n}\left(p_{i}^{p}-\bar{p}_{i}^{p}\right)\left(p_{i}^{o}-\bar{p}_{i}^{o}\right)\right]}{\sqrt{\sum_{i=1}^{n}\left(p_{i}^{p}-\bar{p}_{i}^{p}\right)^{2} \sum_{i=1}^{n}\left(p_{i}^{o}-\bar{p}_{i}^{o}\right)^{2}}}$

$R M S E=\sqrt{\frac{1}{n-1} \sum_{i=1}^{n}\left(p_{i}^{o}-p_{i}^{p}\right)^{2}}$

$S E=\frac{R M S E}{\bar{x}}$

$\operatorname{AIC}(M)=n \cdot \ln \sigma_{z}^{2}+2 M$

تعداد بارامترهاى مدل،

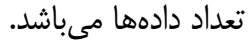

صفر در دو جهت مثبت و منفى در دامنه خود و ميانكين. به به

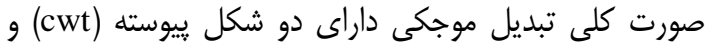

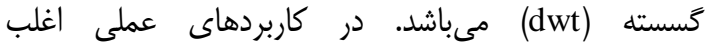

\section{مدلهاي ARIMA}

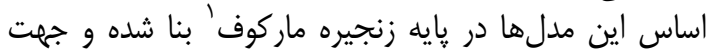

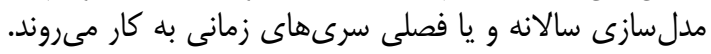

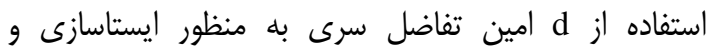

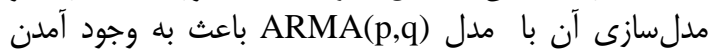

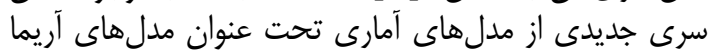
فRIMA(p,d,q)

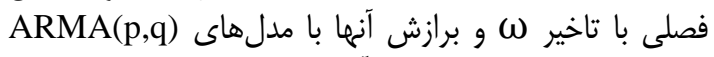

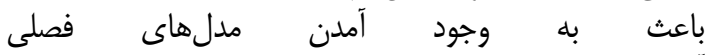

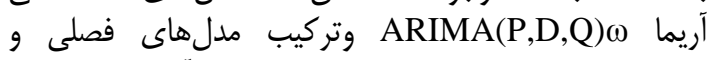

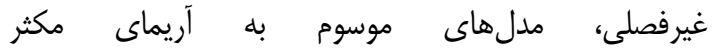

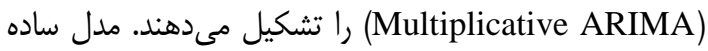

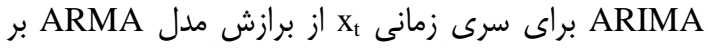
روى سرى تفاضلات آن (un ARIMA به شكل زير خواهد بودا بلات بلات

$$
u_{t}=\sum_{i=1}^{p} \phi_{i} u_{t-i}+\varepsilon_{t}-\sum_{i=1}^{q} \theta_{i} \varepsilon_{t-i}
$$

كه در آن u سرى حاصل حاصل از تفاضل لام سرى اصلى

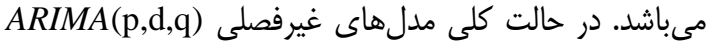
را به صورت زير مىتوان نشان داد:

$\phi(B)(1-B)^{d} z_{t}=\theta(B) \varepsilon_{t}$

كه در آن (B)

$$
\begin{aligned}
& \phi(B)=1-\phi_{1} B-\phi_{2} B^{2}-\cdots-\phi_{P} B^{P} \\
& \theta(B)=1-\theta_{1} B-\theta_{2} B^{2}-\cdots-\theta_{P} B^{P}
\end{aligned}
$$

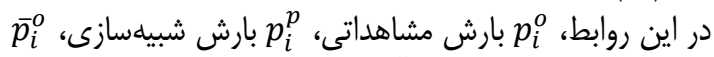
ميانكَين بارش مشاهداتى، 


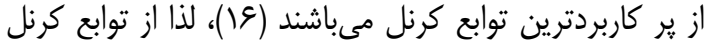

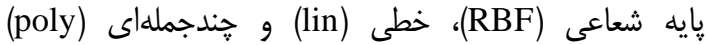
استفاده شد.

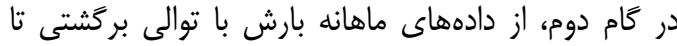

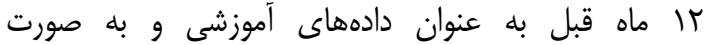

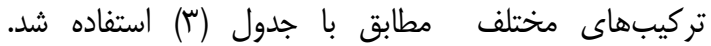

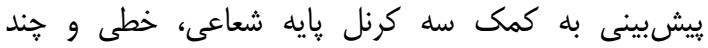

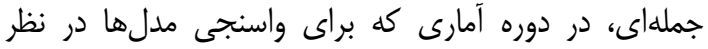

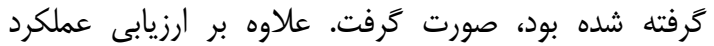

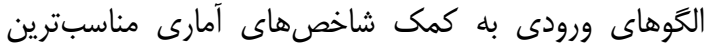

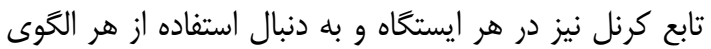

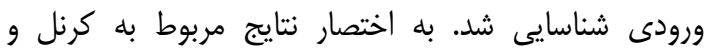

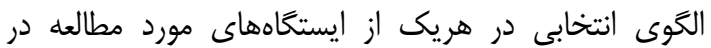
جدول (أ) ارائه شده است.

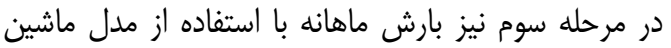

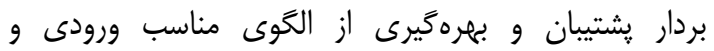

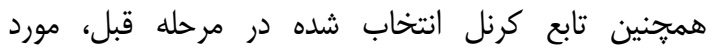

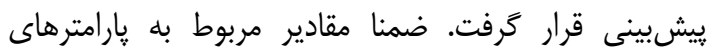

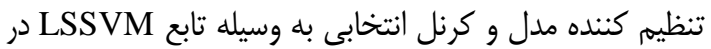

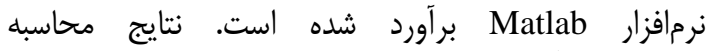
شاخصهاى آمارى در مرحله صحتسنجى در جدول (أ) ارائه

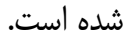

نتايج و بحث

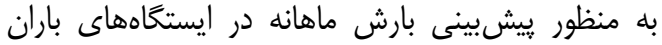

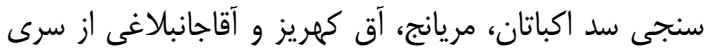

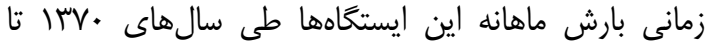

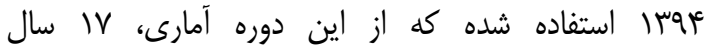

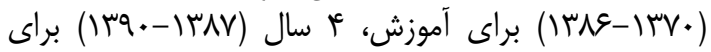

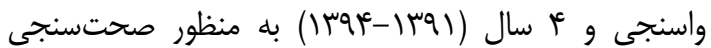

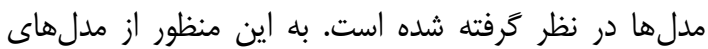

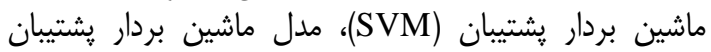

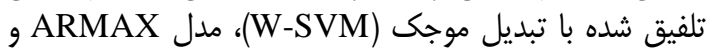

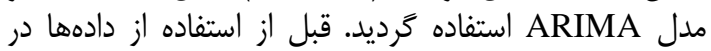

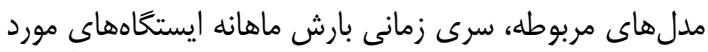

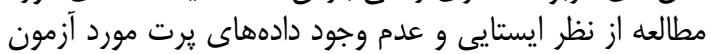

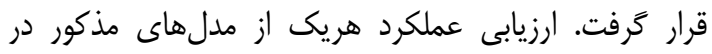

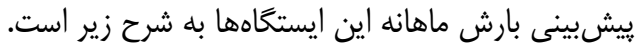

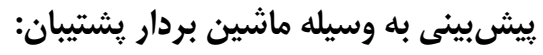

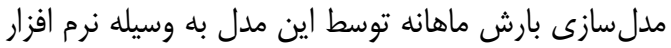

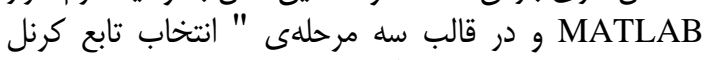

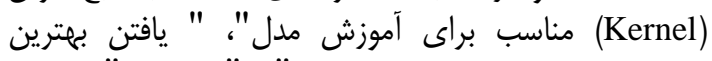

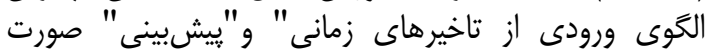

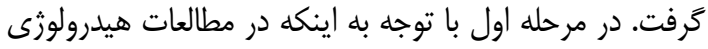

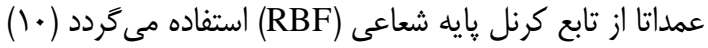

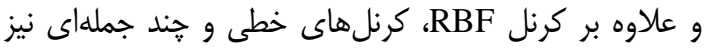

جدول س- الكوى ورودى مدل

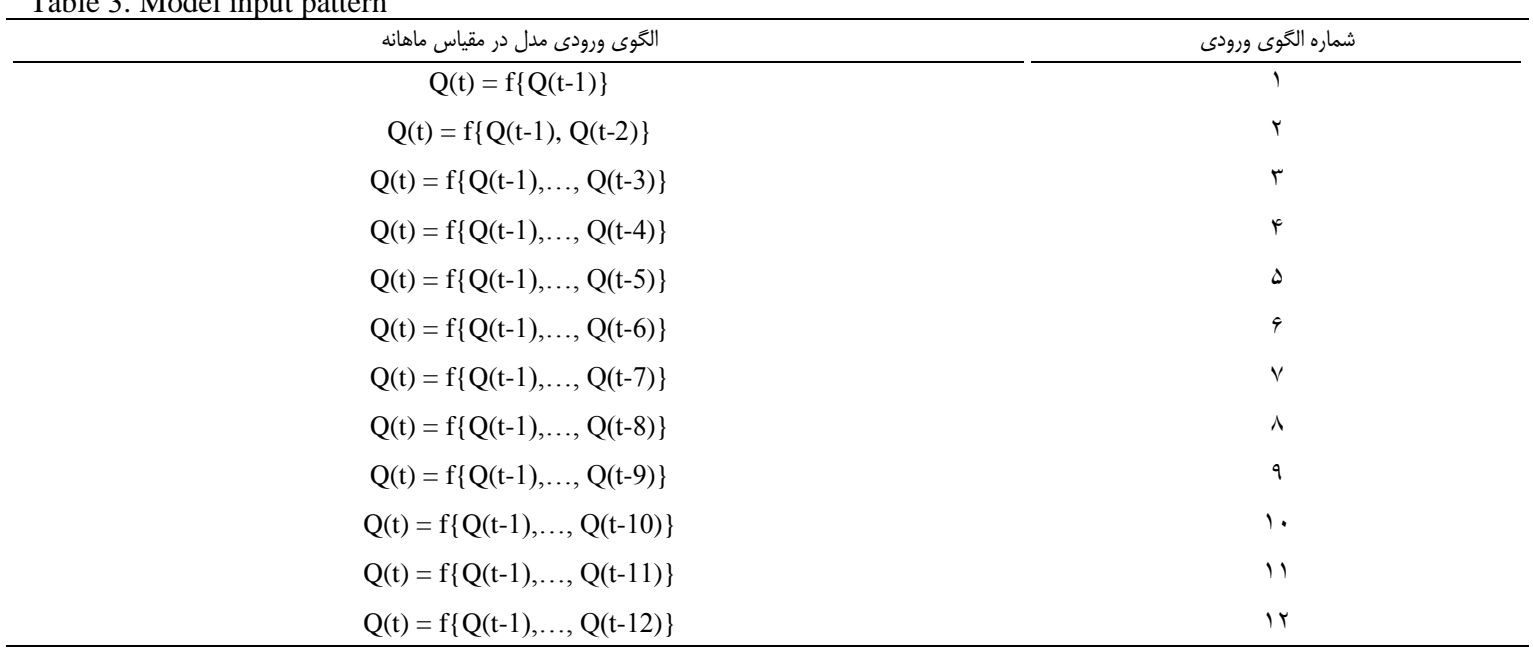


جدول ع - نتايج مربوط به ييشبينى بارش ماهانه در مرحله واسنجى و صحتسنجى به وسيله ماشين بردار ريشتيبان Table 4. The results of monthly precipitation prediction in the calibration and validation step by a support vector machine

\begin{tabular}{|c|c|c|c|c|c|c|c|c|c|}
\hline \multicolumn{3}{|c|}{ مرحله صحت صنجى } & \multicolumn{3}{|c|}{ مرحله واسنجى } & \multicolumn{2}{|c|}{ ساختار مدل } & \multicolumn{2}{|c|}{ ايستخاه } \\
\hline $\begin{array}{l}\mathrm{SE}_{(-)}^{* * *} \\
(-)\end{array}$ & $\begin{array}{l}\mathrm{RMSE}^{* *} \\
\text { (mm/month) }\end{array}$ & $\begin{array}{l}{ }^{*} \\
(-)\end{array}$ & $\begin{array}{l}\mathrm{SE}^{* * * *} \\
(-)\end{array}$ & $\begin{array}{l}\text { RMSE }^{* *} \\
\text { (mm/month) }\end{array}$ & $\begin{array}{l}{ }^{*} \\
(-)\end{array}$ & كرنل & شماره الكو & 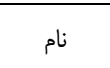 & نوع \\
\hline.$/ 191$ & rE/AVA & $\cdot 19 \cdot 1$ & $1 / 14$ & $r \Delta / v q \Lambda$ &.$/ 91 \mathrm{~V}$ & RBF & it & سد اكباتان & \\
\hline$\cdot / V G V$ & KN/DVE &.$/ 94$. &.$/ 91$. & rV/rqI & $\cdot / \Delta \wedge F^{2}$ & RBF & it & مريانج & \\
\hline I/IQV & KN/VMr &.$/$ tifl & $|/| f \mid$ & Tr/. Fq & $\cdot / F^{\mu} V$ & RBF & it & آق كهريز & \\
\hline.$/ 94 V$ & 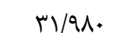 & . /\&MV & $1 / .9$. & $\mathrm{rq} / \Delta \Delta \mathrm{V}$ & - /DHF & RBF & it & آقاجانباغي & \\
\hline
\end{tabular}

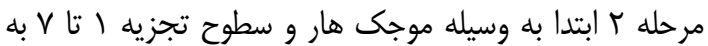

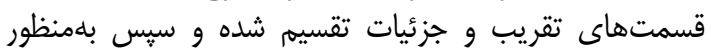

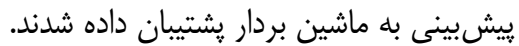

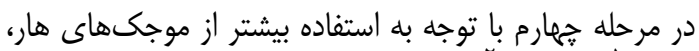

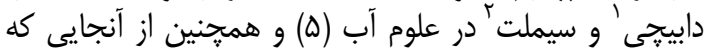

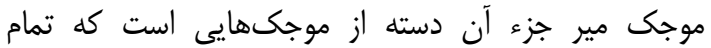

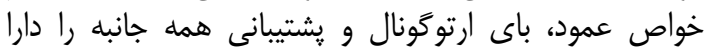

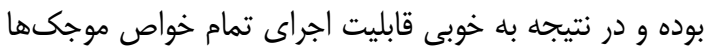

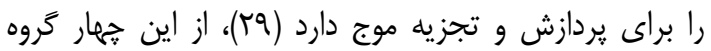

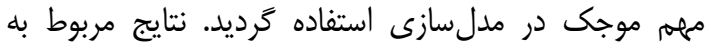

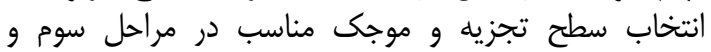

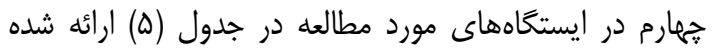

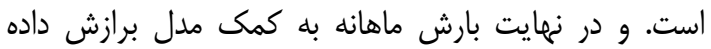

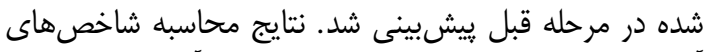

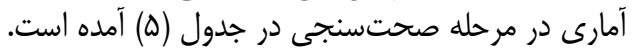

مدل ماشين بردار ريشتيبان (svm) تلفيق شده با تبديل

موجك مدر مان

مدلسازى بارش ماهانه توسط اين مدل به وسيله نرمافزار

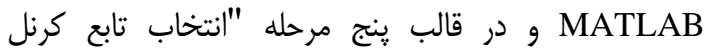

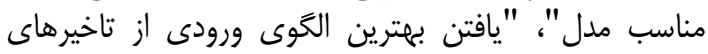

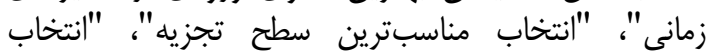

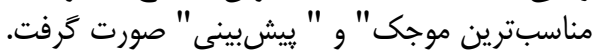

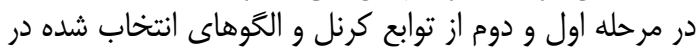

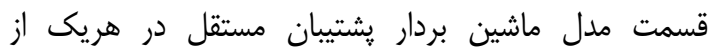
ايستخاههاى مورد مطالعه استفاده گرديد.

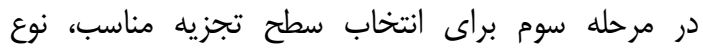

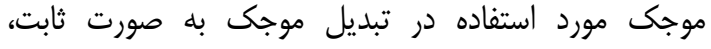
سادهترين نوع موجك كه همان موجك هار (HAAR) است،

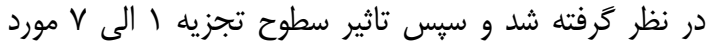
بررسى قرار گرفت. به عبارت ديخر الخوى ورودى سلت انتخابى در

جدول ه- نتايج مربوط به يِشبينى بارش ماهانه در مرحله واسنجى و صحتسنجى به وسيله ماشين بردار يشتيبان تلفيق شده با تبديل موجى

Table 5. The results of monthly precipitation prediction in the calibration and validation step by a support vector machine integrated with wavelet transform

\begin{tabular}{|c|c|c|c|c|c|c|c|c|c|}
\hline \multicolumn{3}{|c|}{ مرحله صحت سنجى } & \multicolumn{3}{|c|}{ مرحله واسنجى } & \multicolumn{2}{|c|}{ ساختار مدل } & \multicolumn{2}{|c|}{ ايستگاه } \\
\hline $\begin{array}{l}\text { SE } \\
(-)\end{array}$ & $\begin{array}{c}\text { RMSE } \\
\text { (mm/month) }\end{array}$ & $\begin{array}{c}\mathrm{r} \\
(-)\end{array}$ & $\begin{array}{l}\text { SE } \\
(-)\end{array}$ & $\begin{array}{c}\text { RMSE } \\
\text { (mm/month) }\end{array}$ & $\begin{array}{c}\mathrm{r} \\
(-)\end{array}$ & سطح تجزيه & موجك & 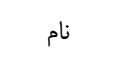 & 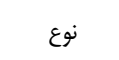 \\
\hline .1 .91 & I/AYF & ./999 & 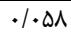 & $\Gamma / . \Delta q$ & ./999 & $r$ & Dmey & سد اكباتان & \\
\hline .1 .40 & 1/99T &.$/ 991$ &.$/ . \Delta F^{F}$ & $r / T \cdot r$ &.$/ 999$ & $r$ & Dmey & مريانج & \\
\hline$\cdot / \cdot \Delta \Delta$ & $1 / T Y \Lambda$ &.$/ 991$ &.$/ . \Delta V$ & $1 / 9 \cdot f^{c}$ &.$/ 999$ & r & Dmey & آق كهريز & • \\
\hline $.1 .9 \mathrm{~V}$ & $r / r \cdot V$ & ./994 & .1 .90 & T/F &.$/ 999$ & $r$ & Dmey & آقاجانبلاغى & \\
\hline
\end{tabular}

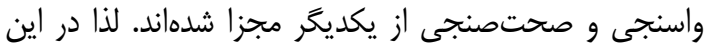

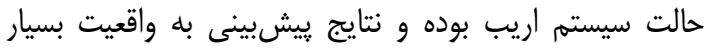

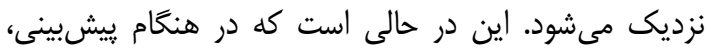

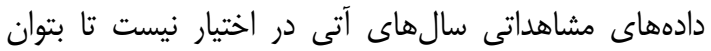

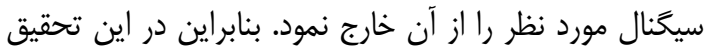

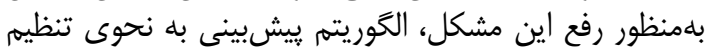

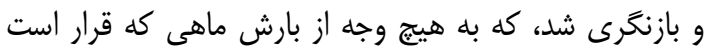

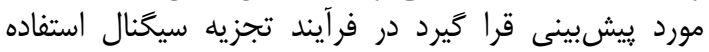

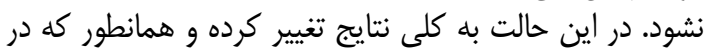

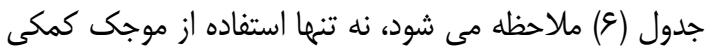

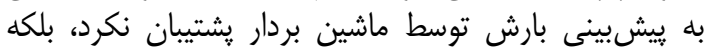

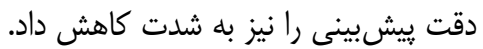

بررسى نتايج در دو مرحله واسنجى و صحتسنجى

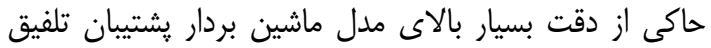

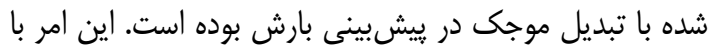

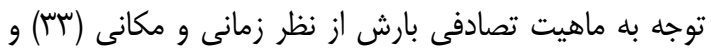

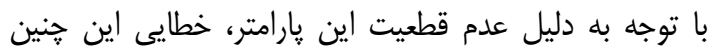

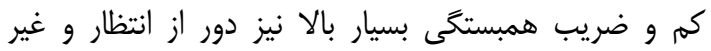

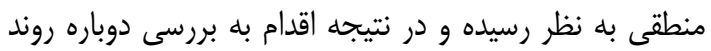

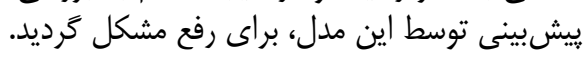

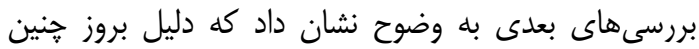

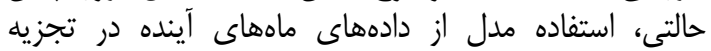

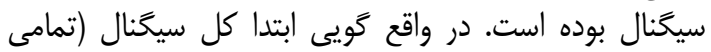

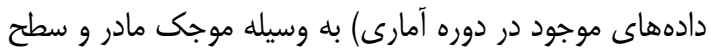

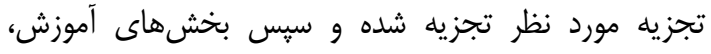


جدول و- نتايج مربوط به ييشيينى بارش در مرحله صحتسنجى به وسيله ماشين بردار يشتيبان تلفيق شده با تبديل موجك اصلاح شده Table 6. Results of precipitation prediction in the validation step by a support vector machine integrated with

\begin{tabular}{|c|c|c|c|c|c|c|}
\hline $\begin{array}{l}\text { SE } \\
(-)\end{array}$ & $\begin{array}{c}\text { RMSE } \\
\text { (mm/month) }\end{array}$ & $\begin{array}{c}r \\
(-)\end{array}$ & سطح تجزيه & موجى & نام ايستخاه & نوع ايستكاه \\
\hline $1 / 4 r q$ & FT/VVD &.$/ 194$ & r & Dmey & سد اكباتان & \multirow{4}{*}{ باران سنجى } \\
\hline $1 / \pi r$. & Fa/FTL & . $/ r T \Delta$ & r & Dmey & مريانج & \\
\hline $1 / \Delta 91$ & rN/vql & $\cdot / 1 \Lambda \mid$ & r & Dmey & آق كهريز & \\
\hline $1 / \subset 90$ & $\Delta \mathrm{D} / \Delta \mathrm{VVA}$ & . & $r$ & Dmey & آقاجانبلاغى & \\
\hline
\end{tabular}

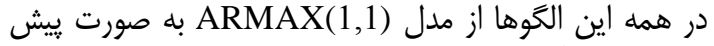

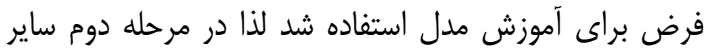

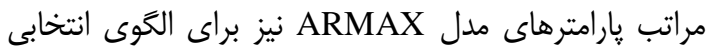

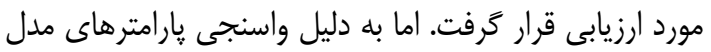

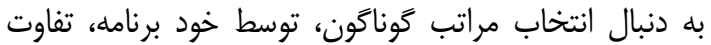

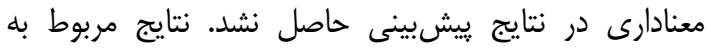

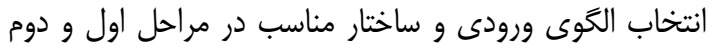

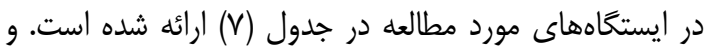

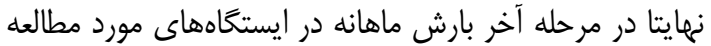

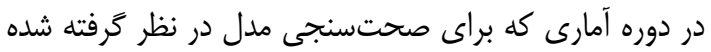

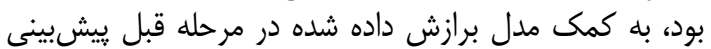

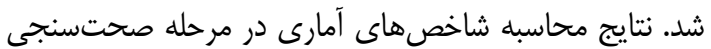
در جدول (V) آمده است.
مدل مدل مدازي بارش مدلسازى بارش ماهانه توسط اين روش، به وسيله مازول

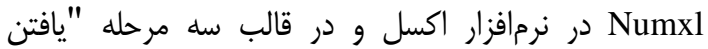
بهترين الخوى ورودى از تاخيرهاى زمانى"، "ئى "يافتن بهترين

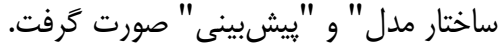

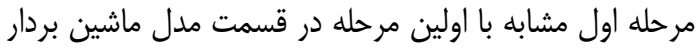

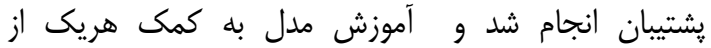

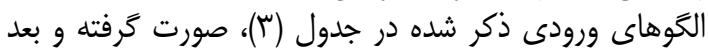

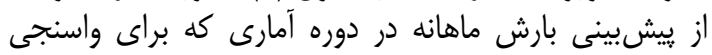

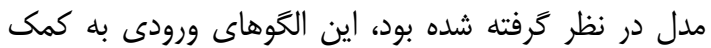

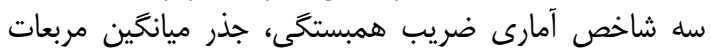

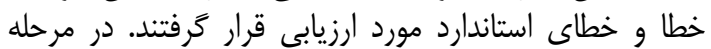

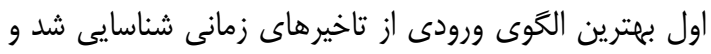

جدول V- نتايج مربوط به يِيشينى بارش در مرحله واسنجى و صحتسنجى به وسيله مدل armax Table 7. Results of precipitation prediction in the calibration and validation step by the armax model

\begin{tabular}{|c|c|c|c|c|c|c|c|c|c|}
\hline \multicolumn{3}{|c|}{ مرحله صحت سنجى } & \multicolumn{3}{|c|}{ مرحله واسنجى } & \multicolumn{2}{|c|}{ ساختار مدل } & \multicolumn{2}{|c|}{ ايستخاه } \\
\hline $\begin{array}{l}\text { SE } \\
(-)\end{array}$ & $\begin{array}{c}\text { RMSE } \\
\text { (mm/month) }\end{array}$ & $\begin{array}{c}\mathrm{r} \\
(-)\end{array}$ & $\begin{array}{l}\text { SE } \\
(-)\end{array}$ & $\begin{array}{c}\text { RMSE } \\
\text { (mm/month) }\end{array}$ & $\begin{array}{c}\mathrm{r} \\
(-)\end{array}$ & $\operatorname{ARMAX}(\mathrm{p}, \mathrm{q})$ & شماره الكَو & 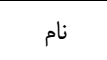 & نوع نوع \\
\hline.$/ 91$. & TV/TFT & $\cdot|\Delta \wedge|$ & $1 / .19$ & r $\Delta / १ \Delta 1$ &.$|9| f$ & $\operatorname{ARMAX}(1,1)$ & $\pi$ & سد اكباتان & \\
\hline - /Arq & $r \cdot / \wedge \Delta q$ & . $/ \Delta S$. & . $/ 989$ & rq/sM &.$/ 498$ & $\operatorname{ARMAX}(1,1)$ & ir & مريانج & \\
\hline $1 / \cdot V V$ & TE/VQV & •/FAV & $1 /$ TNI & rه/৭qF & . & $\operatorname{ARMAX}(1,1)$ & ir & آق كهريز & \\
\hline.$/ 999$ & TN/QVK & $\cdot / \Delta \Delta F$ & $1 / 111$ & $r \cdot / r r q$ & $\cdot 10 .$. & $\operatorname{ARMAX}(1,1)$ & ir & آقاجانبلاغى & \\
\hline
\end{tabular}

در مرحله دوم به منظور بررسى ايستايى سرى زمانى اين

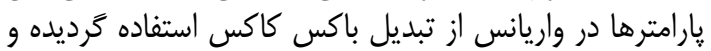

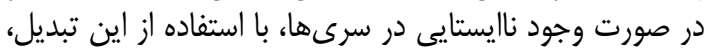
سرى هاى مورد نظر در واريانس ايستا شدند.

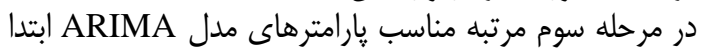

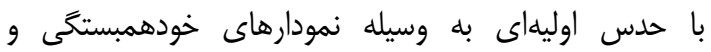

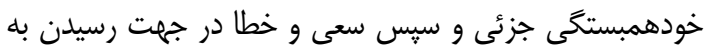

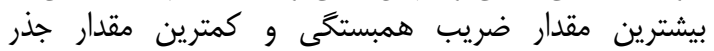

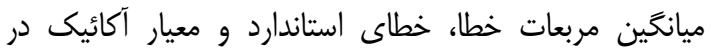

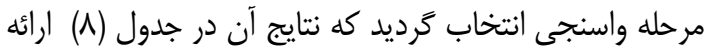
شده است. در مرحله جِهارم جهت تشخيص صحت الكَّى انتخابى،

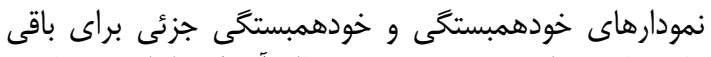

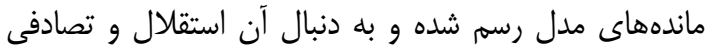

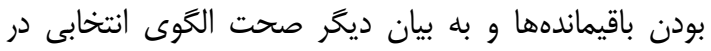

مدل ARIMA

براى اجراى مدل استوكاستيك ARIMA از نرمافزار

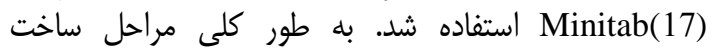

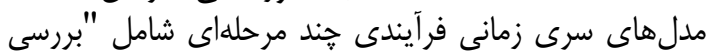

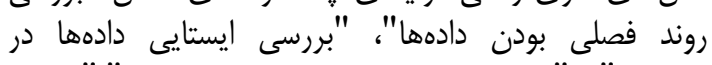

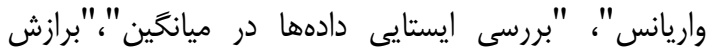

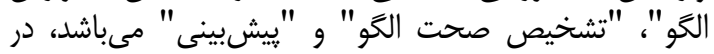

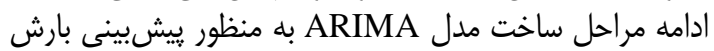
در ايستخاههاى مورد مطالعه تشريح خواهد مدالهد

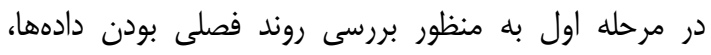

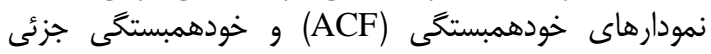
(PACF)

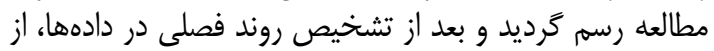

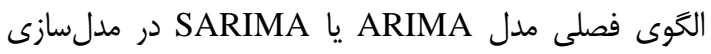
استفاده گرديد. 
صحتسنجى مدل در نظر كرفتله شده بود، مورد بيشبينى

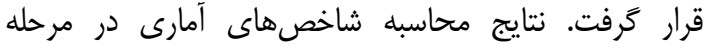
صحتسنجى در جدول (^) آمده است.
مرحله قبل به اثبات رسيد. و نهايتا در مرحله آخر بارش ماهانه

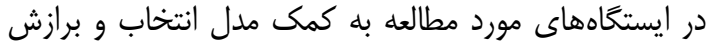

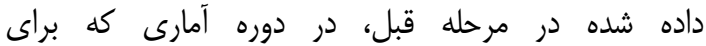

جدول ^- نتايج مربوط به يِيشينى بارش در مرحله واسنجى و صحتسنجى بله وسيله مدل ARIMA Table 8. Results of precipitation prediction in the calibration and validation step by the ARIMA model

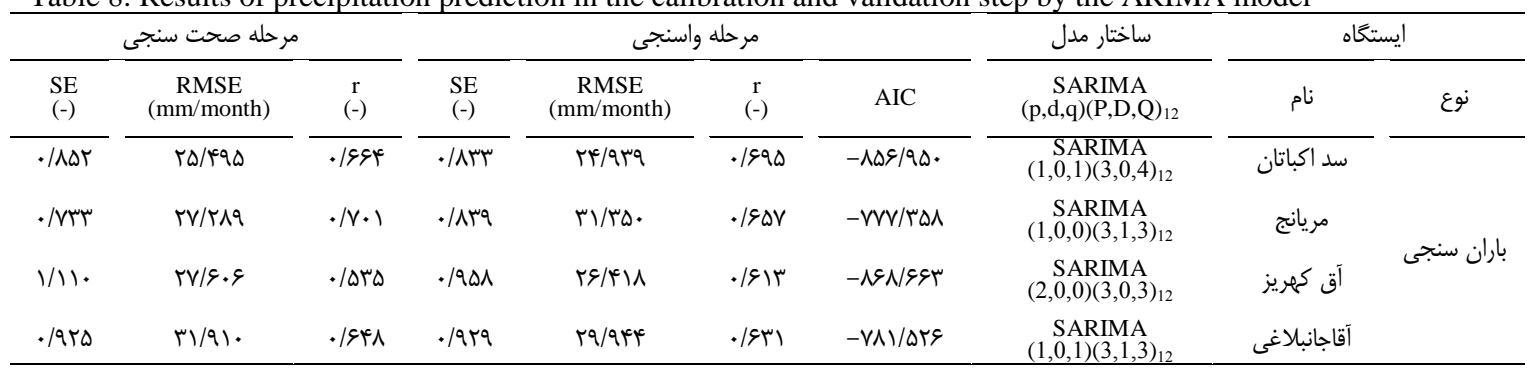

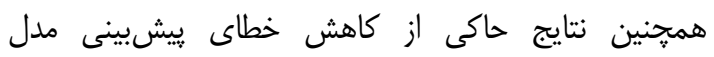
ARIMA نسبت به مدل ARMAX در ايستخاههاى مذكور

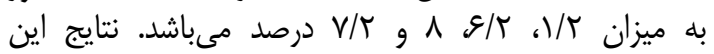

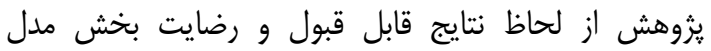

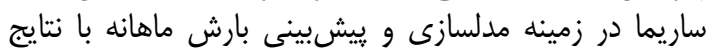

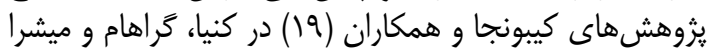

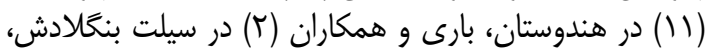

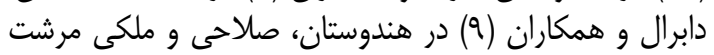

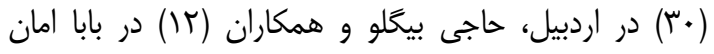

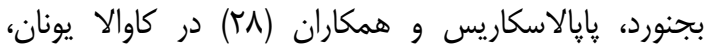

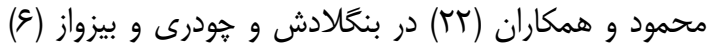

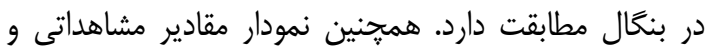

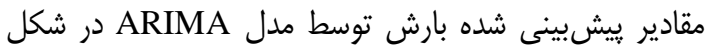
. (r) ائه شده است
جهت مقايسه بهتر، خلاصهاى از نتايج يِيشيينى بارش ماهانه

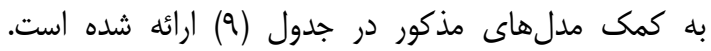

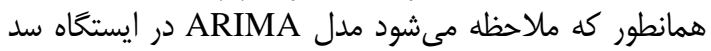
اكباتان با مان

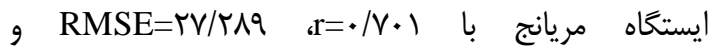

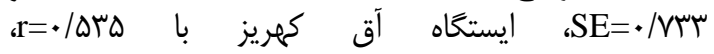
RMSE=TV/\&.9

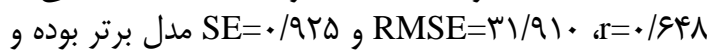

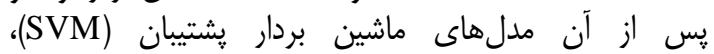
ARMAX

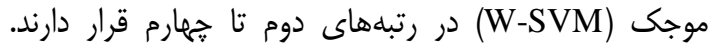

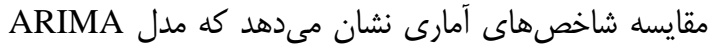

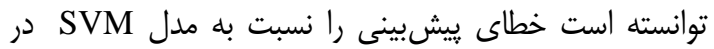

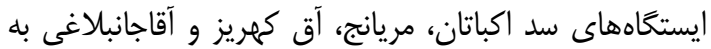

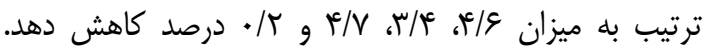

جدول 9- خلاصه نتايج ييشبينى بارش ماهانه در ايستخاههاى مورد مطالعه Table 9. Summary results of Monthly Precipitation prediction at Study Stations

\begin{tabular}{|c|c|c|c|c|c|c|c|c|c|c|c|c|}
\hline \multicolumn{3}{|c|}{ آقاجانبلاغى } & \multicolumn{3}{|c|}{ آق كهريز } & \multicolumn{3}{|c|}{ مريانج } & \multicolumn{3}{|c|}{ سد اكباتان } & مدل \\
\hline ./aTV & rI/qu. & . & $1 / \backslash Q V$ & TN/VTrF & $\cdot / \sin$ & ./VGV & TN/DVE & . $/ 9 T^{\mathrm{C}}$. & ./1८१1 & rE/AVA & $\cdot 19 \cdot 1$ & SVM \\
\hline
\end{tabular}

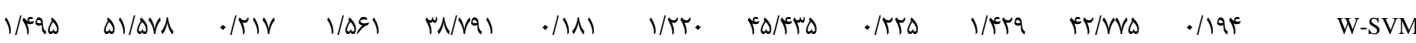

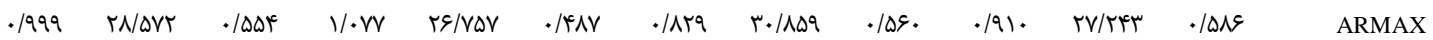

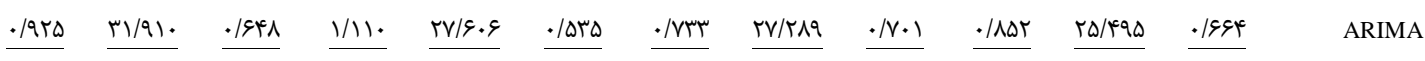



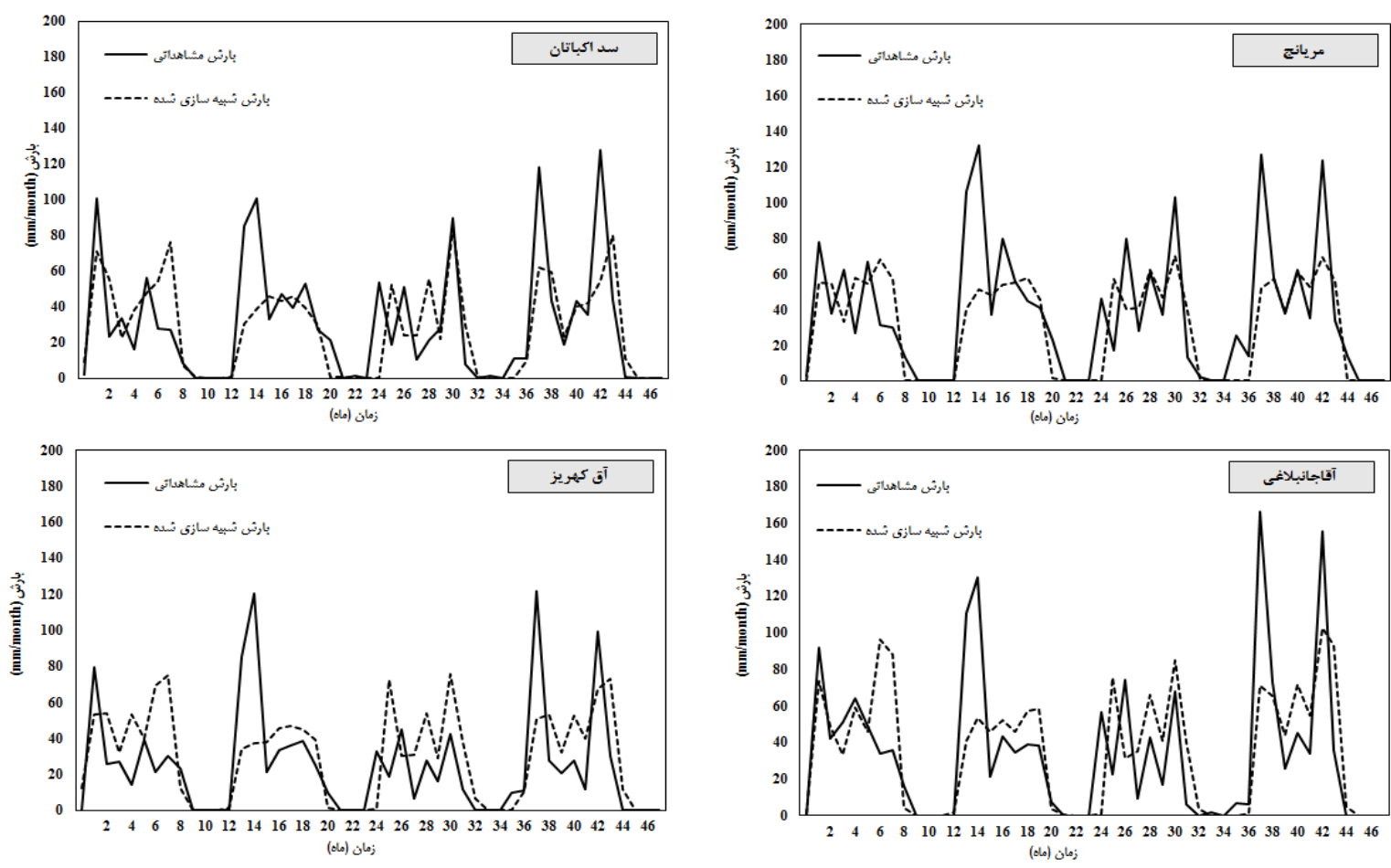

شكل r- مقايسه بارش مشاهداتى و ييشيينى شده توسط مدل ARIMA در مرحله صحتسنجى در ايستخاههاى باران سنجى مورد مطالعه

Figure 2. Comparison of observed and predicted precipitation by ARIMA model at validation step at the studied stations

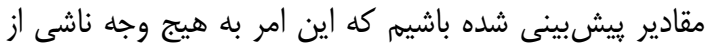

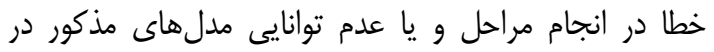

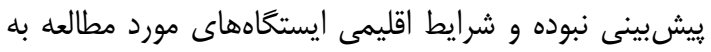

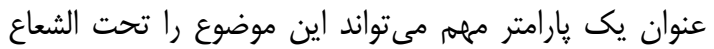

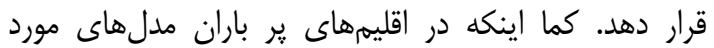

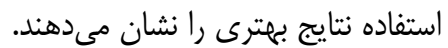

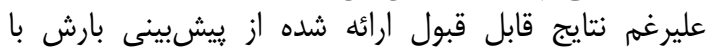

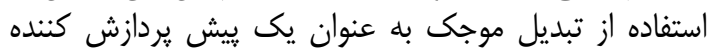

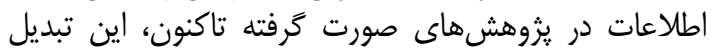

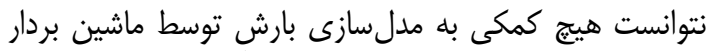

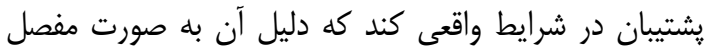

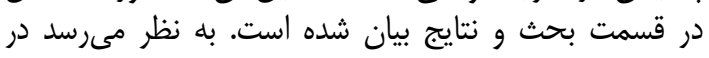

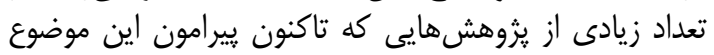

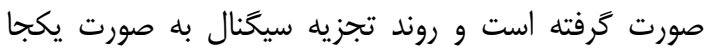

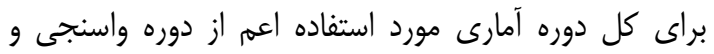

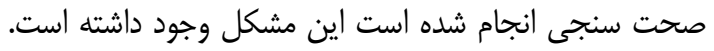

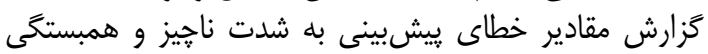

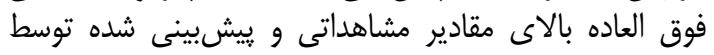

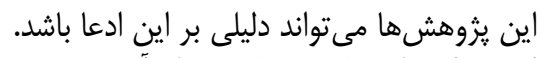

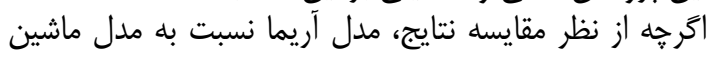

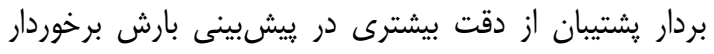

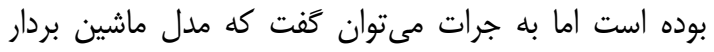

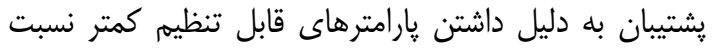

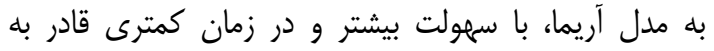

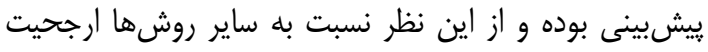
دارد. - مارينا.
به منظور مقايسه دقت و كارايى مدلهاى مذكور در

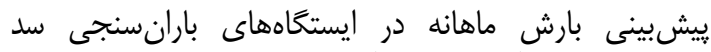

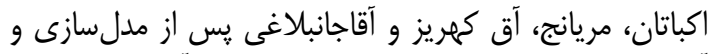

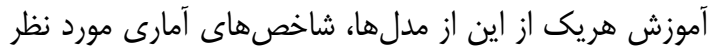

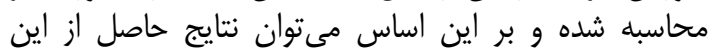
يزوهش، در رابطه با ييشبينى بارش را بانه صورت زير مطرح

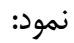
در بين روشهاى ييشبينى مورد مطالعل، در مجموع و درد

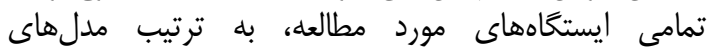

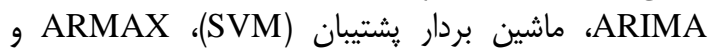
ماشين بردار يشتيبان تلفيق شده با تبديل موجك (W-SVM) در رتبههاى اول تا جهارم قرار دارند.

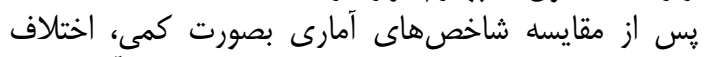

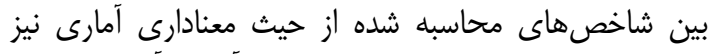

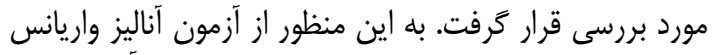

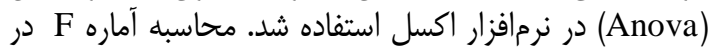

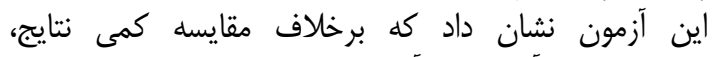

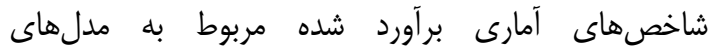

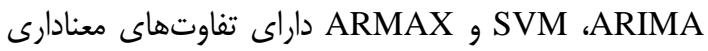

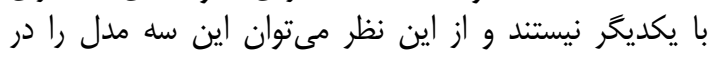

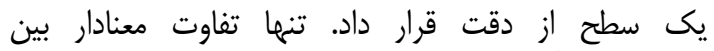

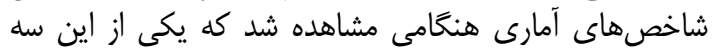

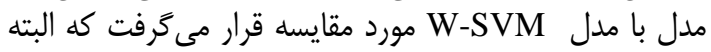

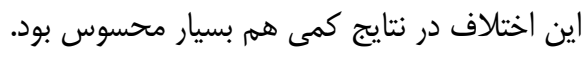

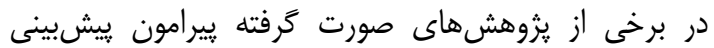

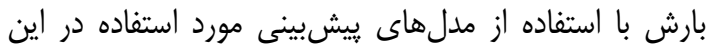

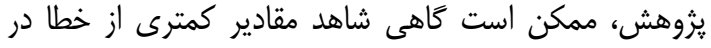


منابع

. Abebe, A.J., D.P. Solomatine and R.G.W. Venneker. 2000. Application of adaptive fuzzy rule-based models for recontruction of missing precipitation events. Hydrological Sciences Journal, 45(3): 425 436.

2. Bari, S.H., M.T. Rahman, M.M. Hussain and S. Ray. 2015. Forecasting Monthly Precipitation in Sylhet City Using ARIMA Model. Civil and Environmental Research, 7(1): 69-77.

3. Behzad, M., K. Asghari and E.A. Coppola. 2010. Comparative Study of SVMs and ANNs in Aquifer Water Level Prediction, Journal of Computing in Civil Engineering, 24(5): 408-413.

4. Cannas, B., A. Fanni and G. Sias. 2005. River flow forecasting using neural networks and wavelet analysis. Geophysical Research Abstracts, Vol. 7, SRef-ID: 1607-7962/gra/EGU05-A-08651.

5. Cannas, B., A. Fanni, L. See and G. Sias. 2006. Data preprocessing for river flow forecasting using neural networks, Wavelet transforms and data partitioning. Physics and Chemistry of the Earth 31(18): 1164-1171.

6. Chowdhury, A. and A. Biswas. 2016. Development of a Monthly Rainfall Prediction Model Using Arima Techniques in Krishnanagar Sub-Division, Nadia District, West Bengal. International Journal of Engineering Studies and Technical Approach, 2(2): 18-26.

7. Coulibaly, F. and N.D. Evora. 2007. Comparison of neural network methods for infilling missing daily weather records. Journal of Hydrology, 341: 27-41.

8. Cristianini, N. and J. Shawe-Taylor. 2000. An Introduction to Support Vector Machines Cambridge University Press, New York, USA.

9. Dabral, P.P., T. Saring and D. Jhajharia. 2016. Time Series Models of Monthly Rainfall and Temperature to Detect Climate Change for Jorhat (Assam), India. Global NEST Journal, 18: 1-14

10. Dehghani, R., M.A. Ghorbani, M. TeshnehLab, A. Rikhtehgar gheasi and E. Asadi. 2015. Comparison and evaluation of bayesian neural network, gene expression programming, support vector machine and multiple linear regression in river discharge estimation (case study: Sufi Chay Basin). Iranian of Irrigation \& Water Engineering, 5(20): 66-85 (In Persian).

11. Graham, A. and E.P. Mishra. 2017. Time series analysis model to forecast rainfall for Allahabad region. Journal of Pharmacognosy and Phytochemistry, 6(5): 1418-1421.

12. Hajibigloo, M., A. Ghazalsoflo and H. Alimirzaee. 2013. Discussion and Forecast Monthly Average Rainfall Techniques Using SARIMA (Case study: Pluviometry Station Babaaman Bojnourd). Journal of Irrigation Science and Engineering, 36(3): 41-54 (In Persian).

13. Hamel, L. 2009. Knowledge Discovery with Support Vector Machines, Hoboken, N.J. John Wiley.

14. Hamidi, O., J. Poorolajal, M. Sadeghifar, H. Abbasi, Z. Maryanaji, H.R. Faridi and L. Tapak. 2014. A comparative study of support vector machines and artificial neural networks for predicting precipitation in Iran. Theoretical and Applied Climatology, 119: 723-731

15. Isazadeh, M., H. Ahmadzadeh and M.A. Ghorbani. 2018. Assessment of Normalization of Monthly Runoff Probabilistic Distribution impact on SVM and ANN Models Performance in Monthly River Flows Simulation (A Case Study: ZarrinehRud River Basin). Journal of Watershed Management Research, 8(16): 22-33 (In Persian).

16. Isazadeh; M., H. Ahmadzadeh and M.A. Ghorbani. 2016. Assessment of kernel functions performance in river flow estimation using support vector machine. Journal of Water and Soil Conservation, 23(3): 69-89 (In Persian).

17. Karamouz, M. and S. Araghinejad. 2005. Advanced Hydrology. $2^{\text {nd }}$ edn., Tehran Polytechnic Press, Tehran, Iran, 464.

18. Khosravi, M. and H. Shakiba. 2010. Precipitation forecasting using artificial neural networks in order to flood management. 4th International Congress of the Islamic Word Geographers (In Persian).

19. Kibunja, H.W., J.M. Kihoro, G.O. Orwa and W.O. Yodah. 2014. Forecasting Precipitation Using SARIMA Model: A Case Study of Mt. Kenya Region. Mathematical Theory and Modeling, 4(11): 5058.

20. Kisi, O. 2009. Neural networks and wavelet conjunction model for intermittent streamflow forecasting. J. of Hydrologic Engineering, 14(8): 773-782.

21. Kisi, O. and M. Cimen. 2012. Precipitation forecasting by using wavelet-support vector machine conjunction model. Engineering Applications of Artificial Intelligence, 25(4): 783-792.

22. Mahmud, I., S.H. Bari and M. Rahman. 2017. Monthly rainfall forecast of Bangladesh using autoregressive integrated moving average method. Environmental Engineering Research, 22(2): 162168.

23. Manzour, D. and M. Yadi Pour. 2016. Studying the Iranian Electricity Market Price with an ARMAXGARCH Mode Quarterly. Journal of Quantitative Economics, 13(1): 97-117.

24. Merry, R.J.E. 2005. Wavelet Theory and Applications. A literature study. Eindhoven University of Technology Department of Mechanical Engineering Control Systems Technology Group. 
25. Mozafari, Gh.A., Sh. Shafiee and H.R. Hemati. 2016. Predicting monthly precipitation of Kermanshah synoptic station using the hybrid model of neural network and wavelet. Journal of Water and Soil Conservation (Journal of Agricultural Sciences and Natural Resources) 22(6): 135152 (In Persian).

26. Najafi, A., S. Azizi Ghalati and M.H. Mokhtari. 2017. Assessment Kernel Support Vector Machines in Classification of Landuses (Case Study: Basin of Cheshmeh kileh-Chalkrod) .Journal of Watershed Management Research, 8(15): 92-101 (In Persian).

27. Omidi, R., F. Radmanesh and H. Zarei. 2014. River flow predicting using stochastic models. The First National Conference on Challenges on Water Resources and Agriculture, Esfahan (In Persian).

28. Papalaskaris, T., T. Panagiotidis and A. Pantrakis. 2016. Stochastic Monthly Rainfall Time Series Analysis, Modeling and Forecasting in Kavala City, Greece, North-Eastern Mediterranean Basin. Procedia Engineering, 162: 254-263.

29. Rostami, M., A. Facheri Fard, M.A. Ghorbani, S. Darbandi and Y. Dinpajoh. 2012. River flow forecasting using wavelet analysis. Irrigation Sciences and Engineering (Jise) (Scientific Journal of Agriculture), 35(2): 73-81 (In Persian).

30. Salahi, B. and R. Maleki Meresht. 2016. Forecasting and Analysis of Monthly Rainfalls in Ardabil Province by Arima, Autoregrressive, and Winters Models. Journal of Water and Soil, 29(5): 13911450 (In Persian).

31. Shafaei, M., J. Adamowski, A. Fakheri-Fard, Y. Dinpashoh and K. Adamowski. 2016. A waveletSARIMA-ANN hybrid model for precipitation forecasting. Journal of Water and Land Development, 28(1): 27-36.

32. Shenify, M., A.S. Danesh, M. Gocić, R. Surya Taher, A.W. Abdul Wahab, A. Gani, S. Shamshirband and D. Petković. 2015. Precipitation Estimation Using Support Vector Machine with Discrete Wavelet Transform. Water Resources Management, 30(2): 641-652.

33. Toufani, P., A. Mosaedi and A. Fakheri Fard. 2011. Prediction of precipitation applying wavelet network model (case study: Zarringol station, Golestan province, Iran). Journal of Water and Soil (Agricultural Sciences and Technology), 25(5): 1217-1226 (In Persian). 


\title{
Evaluation of the Efficiency of Linear and Nonlinear Models in Predicting Monthly Rainfall (Case Study: Hamedan Province)
}

\section{Hamed Nozari ${ }^{1}$ and Fatemeh Tavakoli $^{2}$}

\author{
1- Associate Professor, Water Engineering and Science Department, Bu-Ali Sina University, \\ (Corresponding author: h.nozari@basu.ac.ir) \\ 2- PhD Student Water Engineering and Science Department, Bu-Ali Sina University \\ Received: April 13, $2018 \quad$ Accepted: January 5, 2019
}

\begin{abstract}
In this research, we used the support vector machine (SVM), support vector machine combined with wavelet transform (W-SVM), ARMAX and ARIMA models to predict the monthly values of precipitation. The study considers monthly time series data for precipitation stations located in Hamedan province during a 25-year period (1998-2016). The 25-year simulation period was divided into 17 years for training, 4 years for calibration and 4 years for validation. Statistical comparison of the results was conducted by using correlation coefficient (r), root mean square error (RMSE), and standard error (SE). Results showed that ARIMA, Support Vector Machines, ARMAX and support vector machine combine with wavelet transform were ranked first to forth, respectively. Furthermore, the support vector machine has fewer adjustable parameters than other models. So, the model is able to predict precipitation with greater ease and less time. For this reason, it is preferable to other methods.
\end{abstract}

Keywords: ARIMA, ARMAX, Precipitation, Prediction, Support Vector Machine, Wavelet 